\section{Hydration-Shell Vibrational Spectroscopy}

Dor Ben-Amotz, Purdue University

\section{SUPPORTING INFORMATION}

Vibrational-MCR Methods. Practical applications of vibration-MCR or other spectral decomposition strategies require properly dealing with the rotational ambiguity inherent in all such methods, as well as the suppression of background spectral features that are unrelated to the vibrational spectra of interest. Rotational ambiguity arises from the fact the spectrum of a solution can in general be expressed as a linear combination of the pure solvent spectra and any one of a family of solute-correlated spectra, each of which contain solvent bands with a different (relative) spectral weight. ${ }^{1,2}$ The minimum area solute correlated spectrum (which we refer to as the SC spectrum) is that member of the rotational ambiguity family in which the area of the solvent spectral features are minimized (but remain non-negative). Differences between the shape of the solvent features in this SC spectrum and the pure water spectrum thus highlight solute-induced perturbations in the vibrational spectrum of the solvent.

An additional challenge associated with the quantitative interpretation of vibrational-MCR spectra is that associated with changes in the Raman or IR cross sections of the solute and/or solvent species of interest. For example, although the $\mathrm{OH}$ Raman cross section of water is less sensitive to hydrogen bond strength than the IR absorption cross section, cluster calculations suggest that the Raman cross section of a dangling $\mathrm{OH}$ is about half that of a hydrogen bonded $\mathrm{OH}^{3}$. Moreover, a pi-hydrogen bonded $\mathrm{OH}$ is predicted to have a Raman cross section that is about $20 \%$ larger than an $\mathrm{OH}$ in a water-water hydrogen-bond. ${ }^{4}$ As another example, a recent Raman-MCR host-guest binding study has found that the Raman cross section of benzene increases by about $40 \%$ upon binding to a methylated $\beta$-cyclodextrin cavity. ${ }^{5}$ Additionally, a recent combined Raman-MCR and theoretical study of aqueous $\mathrm{NaOH}$ and $\mathrm{LiOH}$ included predictions that the water symmetric $\mathrm{OH}$ stretch in $\mathrm{H}_{2} \mathrm{O}-\mathrm{Li}^{+}$is about $50 \%$ smaller than that in $\mathrm{H}_{2} \mathrm{O}-\mathrm{Na}^{+}$. ${ }^{6}$ Such cross section changes (as well as rotational ambiguity bounds) must be taken into account in order to obtain quantitative concentration information from measured vibration-MCR SC spectral features.

A practical rule-of-thumb pertaining to the successful application of vibrational-MCR is that obtaining high quality SC spectra typically requires solute concentrations above $\sim 1 \mathrm{wt} \%$, such that at least $\sim 1 \%$ of the solvent molecules are in the first solvation-shell of the solute of interest. It is sometimes possible to obtain reliable $\mathrm{SC}$ spectra from solutions of lower concentration, but only under ideal conditions and in the absence of interfering background signals. In the case of Raman-MCR, the most troublesome interference is often that resulting from fluorescent impurities, which can make it nearly impossible to obtain reliable SC spectra unless the fluorescence is significantly weaker than the Raman spectral features of interest. In the case of IR-MCR, the most significant interfering background can be the interference fringes (etaloning) arising from reflectance by cell windows. Such interference effects are typically absent in attenuated totalinternal reflectance (ATR) spectra, thus making ATR spectra more compatible with IR-MCR analysis than spectra obtained using Fourier-transform infrared spectroscopy (FTIR) or other transmittance-based methods. $^{7,8}$ In all applications of vibrational-MCR it is crucial to correctly establish the background in the SC spectrum in order to assure that the obtained spectrum accurately represents the minimum area SC spectrum, such that any lower area SC spectrum would contain some negative area feature which dips below the baseline. Thus, obtaining a SC spectrum typically requires both establishing a baseline and iteratively adjusting the relative spectral weights (rotational ambiguity) of the pure solvent and solution spectra to obtain a SC spectrum that touches the baseline in some spectral region in which the pure solvent has a significant intensity.

In a two-component system (with a single solute species) the SC solvent bands typically pertain to solvent molecules within the solvation-shell surrounding the solute, as those are the only solvent molecules whose structure differs significantly from the bulk solvent. Important exceptions include systems that are chemically reactive, as well as systems with more than two components. For example, if the solvent is an aqueous $\mathrm{NaOH}$ solution to which acetic acid is added, then the acetic acid SC spectrum will not consist of features arising from acetic acid and aqueous $\mathrm{NaOH}$, but rather will contain features arising both from the acetate ions produced when $\mathrm{OH}^{-}$reacts with $\mathrm{CH}_{3} \mathrm{COOH}$ and from the fact that the solution has a different $\mathrm{pH}$ than the solvent (aqueous $\mathrm{NaOH}$ ), because the solvent itself is chemically modified by the deprotonation of acetic acid. Nevertheless, one may use vibrational-MCR to obtain SC spectra of either acetic acid or sodium acetate in water by adjusting the $\mathrm{pH}$ of the pure $\mathrm{NaOH}$ solution (treated as the solvent in the SMCR decomposition) so as to match that of the solution of interest. ${ }^{1,9}$ Thus, the resulting SC spectra will in general contain only features arising from the solute of interest and local solvent molecules that are perturbed by the solute.

Similarly, one may use the following "head group subtraction" procedure to obtain SC spectra pertaining to the non-polar chain of carboxylic acid (or carboxylate) by including the same concentration of formic acid (or sodium formate) in the SMCR solvent spectrum. ${ }^{9}$ Thus, the resulting $\mathrm{SC}$ spectrum will highlight features pertaining to the addition of a non-polar chain to the carboxylic acid (or acetate) head group. One may use "counter-ion subtraction" procedure to suppress the hydration-shell spectrum of a counter anion such as $\mathrm{Br}^{-}$by including an equimolar concentration of $\mathrm{NaBr}$ in the SMCR solvent spectrum. ${ }^{3,10}$ The success of the latter strategy relies on the fact that the vibrational spectrum of water is minimally perturbed by $\mathrm{Na}^{+}{ }^{11}$

The vibrational-MCR analysis of mixtures containing more than two components requires additional care in resolving local and non-local contributions to SC spectra. For example, when adding a solute to a solvent consisting of an alcohol-water mixture, the SC spectrum may contain features arising from changes in the structure (and composition) of both the local hydration-shell around the 
solute, and the non-local solvent molecules that are far from the solutes. More specifically, such local and nonlocal spectral changes may occur if the solute is non-polar and its solvation-shell has a significantly enhanced alcohol concentration, and thus the surrounding solvent may also have a significantly lower alcohol concentration than the original alcohol-water mixture. ${ }^{12}$

In spite of the challenges associated with the vibrationalMCR analysis of multi-component solutions, sequential two-component SMCR decompositions may provide a robust means of penetrating that complexity. For example, for a three-component mixture, one may perform three separate SMCR analyses to obtain SC spectra of each component, each of which contain intramolecular vibrational features of that component as well as its influence on the vibrational spectra of the other two components. Examples include recent Raman-MCR studies of aqueous solutions containing two alcohols ${ }^{12}$ and various solutes in aqueous salt solutions. ${ }^{13,14}$ Moreover, a two-step SMCR decomposition may be used to quantify aggregation induced spectral changes in aqueous solutions of variable concentration. ${ }^{2,15,16}$ More specifically, an initial SMCR decomposition of pairs of spectra, one of which is pure water and the other is an aqueous solution, may be used to obtain a concentration dependent series of $\mathrm{SC}$ spectra. If all the spectra are essentially identical (when normalized to solute concentration, or the area of some solute intramolecular band) then that implies that there is essentially no coupling between the solutes. Alternatively, as is most often the case, the SC spectra evolve with concentration as a result of an increase in either direct or solvent-separated contacts between solute molecules with increasing solute concentration. One may thus perform a second round of SMCR on the SC spectra in an effort to decompose them into fully hydrated and aggregated spectral components. Such a strategy has been used to quantify water-mediated contributions to solute-solute contact free energies in aqueous solutions, in excess of the contacts that would be present in a random mixture of the same solutes, ${ }^{2,15,16}$ much in the same spirit as the approach taken in the prior isotopic double difference IR study of aqueous acetonitrile. ${ }^{17}$

\section{REFERENCES}

(1) Fega, K. R.; Wilcox, D. S.; Ben-Amotz, D. Application of raman multivariate curve resolution to solvation-shell spectroscopy. Appl. Spectrosc. 2012, 66, 282.

(2) Wilcox, D. S.; Rankin, B. M.; Ben-Amotz, D. Distinguishing aggregation from random mixing in aqueous t-butyl alcohol solutions. Faraday Disc. 2013, 167, 177.

(3) Davis, J. G.; Rankin, B. M.; Gierszal, K. P.; BenAmotz, D. On the cooperative formation of non-hydrogen bonded water at molecular hydrophobic interfaces. Nat. Chem. 2013, 5, 796.

(4) Gierszal, K. P.; Davis, J. G.; Hands, M. D.; Wilcox, D. S.; Slipchenko, L. V.; Ben-Amotz, D. Pi-hydrogen bonding in liquid water. J. Phys. Chem. Lett. 2011, 2, 2930.
(5) Mendes de Oliveira, D.; Ben-Amotz, D. Cavity hydration and competitive binding in methylated $\beta$ cyclodextrin. J. Phys. Chem. Letters 2019, 10, 2802.

(6) Drexler, C. I.; Miller, T. C.; Rogers, B. A.; Li, Y. C.; Daly Jr., C. A.; Yang, T.; Corcelli, S. A.; Cremer, P. S. Counter cations affect transport in aqueous hydroxide solutions with ion specificity. J. Am. Chem. Soc. 2019, 141, 6930 .

(7) Sun, Y. C.; Petersen, P. B. Solvation shell structure of small molecules and proteins by ir-mcr spectroscopy. $J$. Phys. Chem. Lett. 2017, 8, 611.

(8) Daly, C. A. J.; Streacker, L. M.; Sun, Y.; Pattenaude, S. R.; Hassanali, A.; Petersen, P. B.; Corcelli, S. A.; BenAmotz, D. Decomposition of the experimental raman and infrared spectra of acidic water into proton, special pair, and counter-ion contributions. J. Phys. Chem. Lett. 2017, 8,5246 .

(9) Davis, J. G.; Zukowski, S. R.; Rankin, B. M.; BenAmotz, D. Influence of a neighboring charged group on hydrophobic hydration shell structure. J. Phys. Chem. B 2015, 119, 9417-9422.

(10) Long, J. A.; Rankin, B. M.; Ben-Amotz, D. Micelle structure and hydrophobic hydration. J. Am. Chem. Soc. 2015, 137, 10809.

(11) Perera, P. N.; Browder, B.; Ben-Amotz, D. Perturbations of water by alkali halide ions measured using multivariate raman curve resolution. J. Phys. Chem. B 2009, 113, 1805.

(12) Mochizuki, K.; Pattenaude, S. R.; Ben-Amotz, D. Influence of cononsolvency on the aggregation of tertiary butyl alcohol in methanol-water mixtures. J. Am. Chem. Soc. 2016, 138, 9045.

(13) Rankin, B. M.; Ben-Amotz, D. Expulsion of ions from hydrophobic hydration shells. J. Am. Chem. Soc. 2013, 135,8818 .

(14) Vincent, J. C.; Matt, S. M.; Rankin, B. M.; D'Auria, R.; Freites, J. A.; Ben-Amotz, D.; Tobias, D. J. Specific ion interactions with aromatic rings in aqueous solutions: Comparison of molecular dynamics simulations with a thermodynamic solute partitioning model and raman spectroscopy. Chem. Phys. Lett. 2015, 638, 1.

(15) Pattenaude, S. R.; Rankin, B. M.; Mochizuki, K.; BenAmotz, D. Water-mediated aggregation of 2butoxyethanol. Phys. Chem. Chem. Phys. 2016, 18, 24937.

(16) Rankin, B. M.; Ben-Amotz, D.; van der Post, S. T.; Bakker, H. J. Contacts between alcohols in water are random rather than hydrophobic. J. Phys. Chem. Lett. 2015, 6, 688 .

(17) Jamroz, D.; Stangret, J.; Lindgren, J. An infrared spectroscopic study of the preferential solvation in wateracetonitrile mixtures. J. Am. Chem. Soc. 1993, 115, 6165. 